\title{
Two New Species and a Nomenclatural Synopsis of Myriocarpa (Urticaceae) from Mesoamerica
}

\author{
Alexandre K. Monro \\ Department of Botany, The Natural History Museum, London, SW7 5BD, United Kingdom. \\ a.monro@nhm.ac.uk
}

Abstract. Myriocarpa Bentham (Urticaceae), a genus endemic to Central and South America, is characterized by string-like pendent pistillate inflorescences. Among Mesoamerican species, there is significant confusion over the application of names, especially M. bifurca Liebmann, M. cordifolia Liebmann, and $M$. heterospicata Donnell Smith. Two new species from Mesoamerica are described and illustrated: $M$. cubilgueitzensis A. K. Monro and M. trifurca A. K. Monro. In addition, a key to the seven species recognized for Mesoamerica and a nomenclatural review are provided. The names $\boldsymbol{M}$. bifurca Liebmann, $\boldsymbol{M}$. cordifolia Liebmann, and $\boldsymbol{M}$. longipes Liebmann are lectotypified, and the name $M$. colipensis Liebmann is neotypified.

Key words: Guatemala, Mesoamerica, Mexico, Myriocarpa, Urticaceae.

Myriocarpa Bentham is a genus of shrubs and small trees endemic to Central and South America. Within the Urticaceae, Myriocarpa is characterized by its long, pendulous, string-like, spicate, pistillate inflorescences of apparently naked flowers and stems that release a watery latex when cut. Estimates for the size of the genus range from five to 10 (Burger, 1977) to 18 (Friis, 1989) species. Research for Flora Mesoamericana suggests that there are probably 15 to 20 species throughout the range of the genus.

The genus was described by Bentham in 1846 based on Colombian material of Myriocarpa stipitata Bentham collected by Barclay (Barclay, unpublished). Weddell placed Myriocarpa in the Urticaceae tribe Boehmerieae (Weddell, 1856, 1869) despite its anomalous pubescence, cystolith morphology, and wood anatomy. Friis (1989), in his review of the genus's position within the Urticaceae, retained its position within the Boehmerieae, presumably because its position in any other Urticaceae tribe would be equally ambiguous. The position of Myriocarpa within the Boehmerieae is not supported by recent phylogenetic analyses of $\operatorname{trnL}-F$ sequence data (Monro, 2006: fig. 2) that recovered Myriocarpa within a strongly supported clade including both the tribes Urticeae and Lecantheae. Its position within either one of these tribes, however, is unresolved. Based on hair, leaf, and flower morphology, Myriocarpa could equally well be placed in the Lecantheae (absence of hooked hairs) or the Urticeae (alternate leaves, pistillodes not ejecting the achene). Further research, both molecular and morphological, is clearly warranted.

In the last major revision of the genus, six species were recognized (Weddell, 1869). Since this revision, Myriocarpa has attracted little taxonomic interest outside of floristic treatments (e.g., Standley \& Steyermark, 1952; Burger, 1977), despite its unusual pistillate inflorescence morphology and ambiguous position within the Urticaceae. Currently, a total of 24 species names have been published (IPNI, 2006).

\section{Materials and Methods}

In preparing revisionary accounts of Myriocarpa for Flora Mesoamericana, 762 North, Central, and South American collections from BM, C, CAS, CR, DS, F, GH, LAGU, LL, MEXU, MO, NY, PMA, TEX, and US were examined. The macro-morphological characters most frequently used by previous authors have been: leaf shape, pistillate inflorescence branching, orientation of "bracts," and pistil and fruit (achene) morphology. In this study, emphasis was also placed on cystolith arrangement and morphology, stipule morphology, pistillate inflorescence disposition, and staminate flower morphology.

The circumscription of a single character, the fused bract/tepal-like structures located at the base of the perigonium, has remained ambiguous. Bentham (1846) ascribed these to the perigonium and so described them as tepals. Weddell (1856), however, ascribed them to the inflorescence axis and described them as bracts (although in modern terms these would be considered bracteoles). From examination of these structures for the preparation of species accounts for Flora Mesoamericana, they appear independent of the pedicel and to form an envelope around the constricted base of the ovary. The number of these structures associated with each flower $(2,3$, or 4$)$ also corresponds to pistillate tepal numbers frequently encountered in the Urticaceae. In addition, staminate Myriocarpa flowers are subtended by a single 
bracteole at the base of the pedicel, and Myriocarpa would be unique among Urticaceae genera if it had bracteoles associated with both the base and the apex of the pedicel. They are therefore treated as tepals in this treatment.

\section{Taxonomic Treatment and Key to the Mesoamerican SPECIES OF MYRIOCARPA}

Myriocarpa Bentham, Bot. Voy. Sulphur 168, t. 55. 1846. TYPE: Myriocarpa stipitata Bentham, Bot. Voy. Sulphur 168, t. 55. 1846.

Shrubs or trees to $17 \mathrm{~m}$. Stems without stinging hairs, frequently releasing watery latex when cut. Leaves alternate; stipules intrapetiolar, simple, caducous, rarely persistent; blades with margins toothed to subentire; cystoliths fusiform, frequently appearing branched. Inflorescences unisexual; pistillate and staminate inflorescences few-branched racemes or spikes; pedicels, where present, subtended by incon- spicuous bracteoles. Staminate flowers 4-parted, tepals equal, lacking a subapical appendage; pistillode prominent. Pistillate flowers with 2, 3, or 4 equal tepals; stigma foot-shaped. Achene subcompressed, ovoid. Ca. 12 spp., Neotropics.

\section{Key to the Mesoamerican Species of Myriocarpa}

Distributions for each species are given to state level within Mesoamerica. Global distributions are given to regional level (e.g., South America, West Indies) following Flora Mesoamericana protocols (Davidse et al., 1994). Some of the characters in the following keys (e.g., cystoliths) are only visible in dried material. Total flower-bearing branch length is calculated as the sum of the lengths of all the flowerbearing branches within a single inflorescence. Combined with an estimate of flower density, total flower-bearing branch length allows the calculation of the number of flowers in an inflorescence (see below).

\section{Key to Staminate Material}

la. Cystoliths present on abaxial leaf surface.

2a. Leaves subcoriaceous to chartaceous, adaxial surface not minutely pitted, cystoliths randomly scattered throughout, occasionally perpendicular to veins; petiole $3.5-21 \mathrm{~mm} . \ldots \ldots \ldots \ldots \ldots \ldots$. obovata

$2 \mathrm{~b}$. Leaves membranous to subchartaceous, adaxial surface minutely pitted, cystoliths arranged radially around hair bases and/or glands, perpendicular to veins; petiole $13-98 \mathrm{~mm} \ldots \ldots \ldots \ldots \ldots \ldots$. cubilgueitzensis

lb. Cystoliths absent on abaxial leaf surface.

3a. Adaxial leaf surface with cystoliths randomly scattered, minutely pitted occasionally only in part . . 4. M. heterospicata

3b. Adaxial leaf surface with cystoliths arranged radially around hair bases and glands, not minutely pitted.

4a. Plants deciduous; peduncle very densely pubescent, surface completely obscured by hairs which cause it

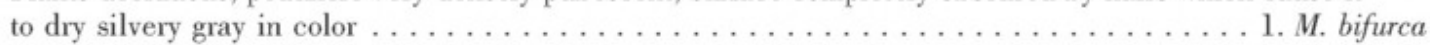

4b. Plants evergreen, rarely deciduous; peduncle glabrous, pubescent, or densely pubescent, the surface visible or completely obscured by the hairs, where completely obscured by hairs these cause it to dry orange-brown in color.

5a. Inflorescence $<90 \mathrm{~mm} \ldots \ldots \ldots \ldots \ldots \ldots \ldots \ldots \ldots \ldots \ldots \ldots \ldots \ldots \ldots \ldots \ldots \ldots \ldots \ldots$ trifurca

5b. Inflorescence $\geq 90 \mathrm{~mm}$.

6a. Flowers $2-2.75 \mathrm{~mm}$ diam. immediately prior to anthesis, stamens $2-3 \mathrm{~mm}$; pedicels $0.13-$ $0.25 \mathrm{~mm} \ldots \ldots \ldots \ldots \ldots \ldots \ldots \ldots \ldots \ldots \ldots \ldots \ldots \ldots \ldots \ldots \ldots \ldots \ldots \ldots \ldots$. cordifolia

6b. Flowers $1.13-2 \mathrm{~mm}$ diam. immediately prior to anthesis, stamens $1.25-1.75 \mathrm{~mm}$; pedicels $0.25-$ $0.75 \mathrm{~mm} \ldots \ldots \ldots \ldots \ldots \ldots \ldots \ldots \ldots \ldots \ldots \ldots \ldots \ldots \ldots \ldots \ldots \ldots \ldots \ldots \ldots$. longipes

\section{Key to Pistillate Material}

1a. Cystoliths present on abaxial leaf surface.

2a. Leaves subcoriaceous to chartaceous, adaxial surface not minutely pitted, cystoliths randomly scattered throughout, occasionally perpendicular to veins; petiole $3.5-21 \mathrm{~mm}$. Inflorescences $11-150 \mathrm{~mm}$. .6. M. obovata

2b. Leaves membranous to subchartaceous, adaxial surface minutely pitted, cystoliths arranged radially around hair bases and/or glands, perpendicular to veins; petiole $13-98 \mathrm{~mm}$. Inflorescences $140-300 \mathrm{~mm}$. . 3. M. cubilgueitzensis

lb. Cystoliths absent on abaxial leaf surface.

3a. Adaxial leaf surface with cystoliths randomly scattered throughout . . . . . . . . . . . . heterospicata

3 b. Adaxial leaf surface with cystoliths arranged radially around hair bases and glands.

4a. Inflorescences coiled or curled in part, especially at the tip, drying very dark brown or almost black to very dark purple, peduncle densely pubescent; plants deciduous................ bifurca

4b. Inflorescences not curled or coiled, drying green, green-brown, pale brown, or red-brown to dark brown, but never dark purple, peduncle sparsely pubescent to pubescent; plants evergreen.

5a. Inflorescence $<2.5 \mathrm{~mm}$ diam., flower density 10 to 36 per $\mathrm{cm} \ldots \ldots \ldots \ldots \ldots$. longipes 5b. Inflorescence $\geq 2.5 \mathrm{~mm}$ diam., flower density 36 to 112 per $\mathrm{cm}$.

6a. Inflorescence 3.5-5.5 mm diam., bearing > 3000 flowers; flower $1.75-2.25 \mathrm{~mm}$. . 2. M. cordifolia 6b. Inflorescence $2.5-3.25 \mathrm{~mm}$ diam., bearing $<2000$ flowers; flower $1.25-1.5 \mathrm{~mm}$. . . 7. M. trifurca 
1. Myriocarpa bifurca Liebmann, Kongel. Danske Vidensk. Selsk. Skr., Naturvidensk. Math. Afd., ser. 5, 2: 307. 1851. TYPE: Nicaragua. [Masaya:] in Monte Masaija, s.d., A. S. Oersted 5850 ["I2/ 47 '] (lectotype, designated here, C 100/99 2).

Myriocarpa malacophylla B. L. Robinson \& Bartlett, Proc. Amer. Acad. Arts 43: 50. 1907. TYPE: Guatemala. Zacapa: Gualán, 12 Jan. 1905, C. C. Deam 361 (holotype, GH).

Discussion. There are two sheets of the type collection at $\mathrm{C}$, both have been numbered Oersted 5850 . However, they appear to have been collected in different months, one sheet being annotated "12/47" and the other "11/47," and therefore probably represent different collections. The sheet annotated "12/47" has been selected as lectotype.

Habitat and distribution. Myriocarpa bifurca is found in disturbed and secondary deciduous broadleaf forest, riparian vegetation, and shade coffee farms, at elevations of $100-1300 \mathrm{~m}$ and is distributed from Mexico to Costa Rica (see below).

Selected specimens examined. COSTA RICA. [s. loc.]: Brenes 6672 (F, NY), Téllez 4289 (MEXU). Alajuela: Hammel 18751 (BM). Guanacaste: Chavarría 906 (CR), Flores \& Herrera 65 (MO), Liesner 4968 (CR, MO). Puntarenas: Bello 4386 (CR), Burger 3911 (F, MEXU, NY), Haber \& Zuchowski 9750 (BM, CR, F), Haber \& Zuchowski 10538 (BM), Haber \& Zuchowski 10471 (MO), Haber \& Zuchowshi 10490 (BM), Haber \& Zuchowski 10459 (BM). EL SALVADOR. [s. loc.]: Seiler 1179 (F). Ahuachapán: Monro et al. 1901 (BM), Sandoval et al. s.n. [JBL839] (F), Sermeno s.n. [JBL 1033] (BM, LAGU, MO), Standley 19937 (NY, US). Cabañas: Davidse et al. 37073 (BM). La Libertad: Calderón \& Berendsohn s.n. [JBL569] (MO), Carlson 72 (F), Williams \& Molina R. 15248 (F), Cruz \& Berendsohn 458 (BM, LAGU), Monro et al. 2295 (BM), Montalvo \& Villacorta 6459 (F), Sidwell et al. 473 (BM), Standley 19148 (NY). Morazán: Calderón 1951 (US). San Miguel: Monro et al. 2078 (BM), Tucker 966 (F, LL, NY). San Salvador: Sidwell et al. 600 (BM). San Vicente: Standley 21671 (NY). Santa Ana: Harmon \& Fuentes 4957 (MO). GUATEMALA. [s. loc.]: Williams et al. 23784 (F). Huehuetenango: Castillo C. \& Castillo 2164 (C, F). Jalapa: Kellerman 6454 (F, US). Quetzaltenango: Steyermark 33833 (F). Retalhuleu: Standley 88131 (US), Steyermark 88154 (F). Santa Rosa: Kellerman 7758 (MEXU, US). Zacapa: Steyermark 42725 (F, US). HONDURAS. [s. loc.]: Allen 6464 (F, PMA). Francisco Morazán: Molina R. 792 (F), Nelson \& Vargas 3479 (MO). Santa Bárbara: Standley \& Lindelie 7538 (F). MEXICO. Chiapas: Breedlove 30626 (MEXU, MO), Breedlove 23945 (MO), Breedlove \& Thorne 30386 (MEXU), Langman 3814 (US), Laughlin 2995 (F, MEXU), Matuda 5476 (LL, MEXU), Matuda 18750 (F), Palacios E. 251 (MEXU), Palacios E. 308 (MEXU), Reyes G. et al. 1561 (BM), Reyes G. et al. 1693 (BM). Oaxaca: Martinez R. 329 (BM, MEXU). NICARAGUA. Estelí: Krukoff \& Stevens 5765 (BM, MEXU, MO), Moreno 25324 (BM, MO), Moreno 15316 (BM, MEXU), Moreno 19935 (MO), Moreno 14054 (BM, MO), Moreno 19967 (BM, MO). Granada: Moreno 6322 (MO), Moreno 6365 (BM, MO), Moreno 419 (BM, MO). Jinotega: Araquistain \& Moreno
1625 (BM, MO). Madriz: Stevens et al. 16033 (MO). Managua: Croat 39054 (MO), Garnier A. 504 (F, US), Sandino 310 (BM, MO), Standley 8363 (F). Masaya: Araquistain \& Moreno 589 (BM, MO), Moreno 6139 (BM, MO), Moreno \& López 7174 (MO), Neill 3071 (BM, MO), Oersted 21722 (MO photo ex B, F photo ex B), Oersted 5850 (C, MO, US), Seymour 3308 (F), Stevens \& Krukoff 5261 (BM, MEXU, MO). Matagalpa: Moreno 22625 (BM, MO). Nueva Segovia: Moreno 19448 (MO), Moreno 13727 (MO).

2. Myriocarpa cordifolia Liebmann, Kongel. Danske Vidensk. Selsk. Skr., Naturvidensk. Math. Afd., ser. 5, 2: 306. 1851. TYPE: Mexico. Veracruz: Colipa, s.d., F. M. Liebmann 14226 (lectotype, designated here, C 100/99 4; isotypes, F fragm. at US).

Myriocarpa colipensis Liebmann, Kongel. Danske Vidensk. Selsk. Skr., Naturvidensk. Math. Afd., ser. 5, 2: 306. 1851. TYPE: Mexico. Veracruz: Mun. de Coatzintla, Palmar de Zapata, 110 m, 25 Jan. 1982, Cortés V. 107 (neotype, designated here, NY; isotype, MEXU not seen).

Myriocarpa brachystachys S. Watson, Proc. Amer. Acad. Arts 26: 152. 1891. TYPE: Mexico. Jalisco: barranca, near Guadalajara, 21 May 1889, C. G. Pringle 3024 (holotype, US not seen; isotypes, NY, VT not seen).

Discussion. The collection selected as lectotype for Myriocarpa cordifolia was designated a lectotype through an annotation by Dennis Woodland (in sched.) in 1984. The collection selected as lectotype was made on the basis that it was cited by Liebmann in the original species description, that the sheet selected had been annotated by Liebmann, and that this was the duplicate with the most fertile material.

No type specimen was cited in the original description of Myriocarpa colipensis, and no material annotated by Liebmann with this epithet and locality has been located at $\mathrm{C}$ or elsewhere. For this reason, a neotype has been designated. This collection was selected because it includes pistillate inflorescences, is in a good state of preservation, and originates from the same state in Mexico (Veracruz) as the locality given for this species by Liebmann (Colipa).

Habitat and distribution. Myriocarpa cordifolia is found in riparian vegetation; disturbed and undisturbed wet tropical-, montane-, and cloud-forest; and oak-pine-liquid amber forest formations, from sea level to $2500 \mathrm{~m}$, from Mexico to Costa Rica.

Selected specimens examined. COSTA RICA. [s. loc.]: Leon 546 (F, MO), Skutch 3521 (NY), Skutch 3527 (US), Alajuela: Smith 1553 (NY). Cartago: Barringer et al. 2262 (CR, F, LL), Utley \& Utley 2132 (F, LL). Heredia: Garwood et al. 392 (BM, F, MEXU), Gomez P. 2156 (F), McDowell 934 (F, TEX). Puntarenas: Bello 709 (BM, CR, MO), Haber \& Zuchowski 9805 (MO). San José: González E. 158 (F), Grayum \& Stiles 5309 (BM, CR, MEXU, MO), Maas \& Hammel 7949 (BM, CR, F, MO), Standley 36488 (US), 
Standley 37819 (US), Taylor 17928 (NY, US), Utley \& Utley 626 (MO), Wilbur 24466 (MO). GUATEMALA. El Quiche: Contreras 5202 (F, LL), Proctor 25390 (F, LL). Huehuetenango: Breedlove 11677 (LL). Santa Rosa: Standley 79344 (F). MEXICO. Chiapas: Breedlove 49659 (MEXU), Breedlove 9415 (F), Matuda 278 (MEXU), Méndez T. 5305 (MEXU), Shilom T. 2088 (NY), Breedlove 8966 (F), Shilom T. 3976 (LL), González G. 219 (TEX), Reyes G. et al. 1605 (BM, MEXU), Xolocotzi \& Sharp X-469 (MEXU). Jalisco: Pringle 4735 (BM), Pringle 3024 (NY). Michoacán: Labat 1601 (MEXU). Temasealtepec: Hinton et al. 3780 (BM). Veracruz: Cortés V. 107 (NY), Liebmann 14226 (C, F, US), Nee et al. 29714 (BM, F, MO, NY, TEX, XAL), Nee \& Calzada 22680 (NY), Ventura A. 4755 (TEX).

3. Myriocarpa cubilgueitzensis A. K. Monro, sp. nov. TYPE: Guatemala. Alta Verapaz: pantanó, 2.5 mi. W of Cubilgüitz, 250-300 m, 28 Feb. 1942, J. A. Steyermark 44346 (holotype, US). Figure 1.

Species nova Myriocarpae obovatae Donnell Smith similis, sed ab ea foliis membranaceis subchartaceisve supra minute foveolatis atque petiolo saepe longiore, cystolithis radialiter dispositis et inflorescentiae pistillatae ramulis majoribus differt.

Shrub or tree to $9 \mathrm{~m}$, evergreen. Young shoots pubescent to sparsely pubescent, the hairs 0.25 $0.5 \mathrm{~mm}$, erect or appressed, weakly curved, straight, or crooked; internodes 6-25 × 2.5-4 mm. Stipules 5$11 \mathrm{~mm}$; petioles $13-98 \times 1-2 \mathrm{~mm}$, petioles of younger leaves pubescent, becoming glabrous, the hairs $0.5-0.75 \mathrm{~mm}$; leaf blade $80-206 \times 32-79 \mathrm{~mm}$, obovate, rhombic, oblanceolate, or ovate, membranous to subchartaceous; adaxial surface minutely pitted, glabrous, the cystoliths fusiform and V-shaped fusiform, arranged radially around hair bases and/or glands, perpendicular to veins; abaxial surface sparsely pubescent to glabrous, the hairs when present ca. $0.75 \mathrm{~mm}$, appressed, weakly appressed, or erect, weakly curved, straight, or crooked, cystoliths fusiform, V-shaped fusiform, and X-shaped fusiform, arranged radially around hair bases and/or glands, perpendicular to veins; principal veins 3 , lateral principal veins visible for $1 / 2-2 / 3$ of leaf length, secondary veins 2 to 4 pairs, forming a $30^{\circ}-60^{\circ}$ angle with the midrib, domatia absent; base acute or obtuse to cuneate; margins serrate toward apex, basal 1/10 $1 / 5$ entire; apex cuspidate or subcuspidate. Staminate inflorescences 20-110 mm, a single order of branching, with a total flower-bearing branch length of 15$122 \mathrm{~mm}$; peduncular bracts $2-2.5 \mathrm{~mm}$; peduncle glabrous to sparsely pubescent, the hairs 0.25 $0.375 \mathrm{~mm}$; flowers sessile to subsessile. Pistillate inflorescences 1 to 5 per stem, pendent, $140-300 \times$ $3-4 \mathrm{~mm}$, bearing 1083 to 2950 flowers, pale greengray when fresh, pale brown when dry, dichotomously and/or trichotomously branched 1 or $2 \times$, with a total flower-bearing branch length of $550-770 \mathrm{~mm}$ and flower density of 16 to 50 per $\mathrm{cm}$; peduncular bract ca. $1 \mathrm{~mm}$; peduncle $11-30 \mathrm{~mm}$, sparsely pubescent to glabrous, when pubescent the hairs ca. $0.25 \mathrm{~mm}$, erect, straight; flowers pedicellate to subsessile, pedicels where present $0.13-0.25 \mathrm{~mm}$. Staminate flowers $0.75-1 \times 1.5-2 \mathrm{~mm}$ immediately prior to anthesis, tepals $1.5-1.75 \mathrm{~mm}$; stamens ca. $2 \mathrm{~mm}$. Pistillate flowers/fruit $1.75-2.5 \times 0.68-1 \mathrm{~mm}$; tepals 2, 0.75-1 mm, appressed; ovary $0.75-1 \mathrm{~mm}$, ellipsoid, constricted from base for 1/3-1/2 of length, sparsely to moderately pubescent, the hairs $0.25-$ $0.5 \mathrm{~mm}$; style $0.5-0.75 \mathrm{~mm}$; stigma very strongly asymmetrical.

Habitat and distribution. Myriocarpa cubilgueitzensis is found in lowland forest in rocky areas and in riparian vegetation. It is distributed in northern Mesoamerica, Mexico (Chiapas, Oaxaca, Tabasco, Veracruz), and Guatemala (Alta Verapaz) at elevations of $130-600 \mathrm{~m}$.

IUCN Red List category. Conservation for Myriocarpa cubilgueitzensis must be considered as Least Concern (LC) according to IUCN Red List criteria (IUCN, 2001), owing to the fact that the species has been collected 11 times in several localities in Mexico and Guatemala.

Etymology. The species is named after the locality of the type collection.

Discussion. Myriocarpa cubilgueitzensis most closely resembles $M$. obovata Donnell Smith in the presence of cystoliths on the abaxial leaf surface (these unique to these two species) and the pale green-gray (when fresh), strongly bilaterally compressed pistillate flowers. Material of this species has frequently been determined as $M$. heterospicata Donnell Smith. The three species may be distinguished from each other as follows: (1) in $M$. cubilgueitzensis, cystoliths present on abaxial leaf surface, adaxial surface minutely pitted, cystoliths arranged radially around hair bases and/or glands, perpendicular to veins; leaves membranous to subchartaceous; petiole 13-98 mm; pistillate inflorescence 140-300 mm; (2) in M. heterospicata, cystoliths absent from abaxial leaf surface, adaxial surface minutely pitted, cystoliths randomly scattered throughout or occasionally radially around hair bases and/or glands; leaves chartaceous; petiole 6.5$125 \mathrm{~mm}$; pistillate inflorescences $62-330 \mathrm{~mm}$; and (3) in M. obovata, cystoliths present on abaxial leaf surface, adaxial surface not minutely pitted, cystoliths randomly scattered throughout, occasionally perpendicular to veins; leaves subcoriaceous to chartaceous; 


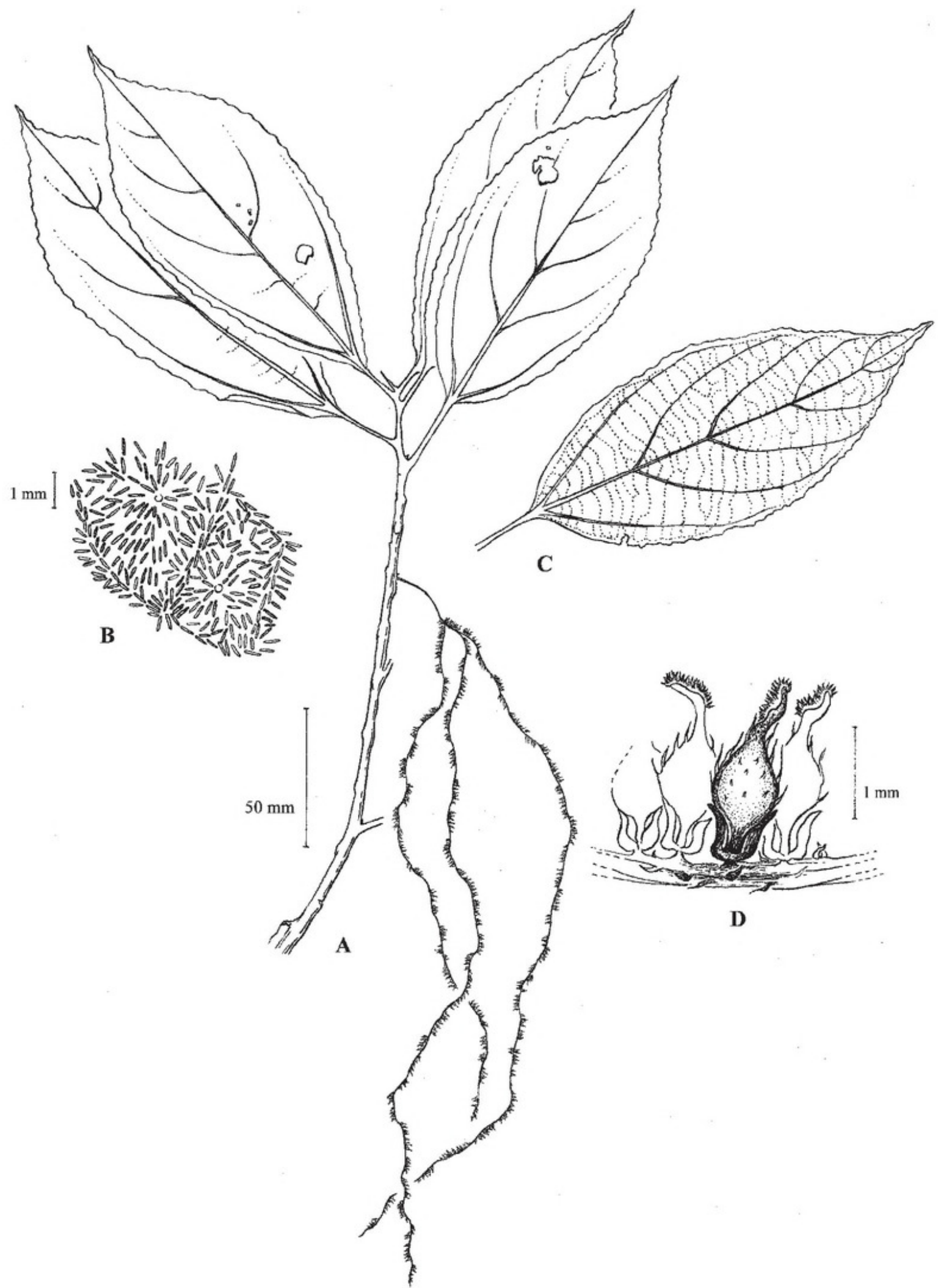

Figure 1. Myriocarpa cubilgueitzensis A. K. Monro. - A. Habit, with pistillate inflorescence. -B. Cystolith arrangement of abaxial leaf surface. - C. Leaf, abaxial surface. -D. Pistillate flowers. A-D drawn from the holotype, J. A. Steyermark 44346 (US). 
petiole 3.5-21 mm; pistillate inflorescence 11$150 \mathrm{~mm}$.

Paratypes. GUATEMALA. Alta Verapaz: Cubilgüitz, H. von Tuerchheim 7982 (NY, US); above slopes of waterfall, along Río Icvolay, N \& NW of Finca Cubilgüitz to Quebrada Diablo, 300-350 m, 6 Mar. 1942, J. A. Steyermark 44778 (F, US). MEXICO. Chiapas: Mun. Ocosingo, M. Martínez-Icó 361 (MEXU). Oaxaea: Chimalapa, Mun. Santa María Chimalapa, Piedra Blanca (Popotzá), al S de la vereda al Río Blanca ca. $7 \mathrm{~km}$ al E de Santa María, H. Hernández $G$. 745 (LL); Distr. de Ixtlán, Mun. Yolox along rd. betw. Yolox \& Comâltepec at river crossing closest to Yolox, G. J. Martin 405 (BM). Tabasco: Mun. Teapa, Sierra El Madrigal, a 600 E del edificio principal del Centro Regional Tropical Puyacatengo, A. M. Hanan A. 607 (MEXU). Veracruz: Mun. Hidalgotitlán, camino campamiento hermanos CedilloPlan de Arroyo, J. Dorantes \& C. Castillo 3628 (TEX, XAL not seen); Mun. Minatitlán, 12 km NE de Uxpanapa (Poblado 12) sobre camino a Díaz Ordaz, loma NW del Poblado 13, $T$. Wendt, A. Villalobos C. \& I. Navarrete 3695 (MO); Mun. Minatitlán, lomas al S del Poblado 11, ca. 27 km E de La Laguna, cima de una loma al NNE de la loma grande, $T$. Wendt, A. Villalobos C. \& I. Navarrete 3127 (MO); Mun. Minatitlán, $6.4 \mathrm{~km} \mathrm{~N}$ de la terracería La Laguna-Uxpanapa sobre el camino nuevo brecha 93 a Belisario Domínguez, $T$. Wendt, A. Villalobos C. \& I. Navarrete 4281 (MO).

4. Myriocarpa heterospicata Donnell Smith, Bot. Gaz. (Crawfordsville) 12: 133. 1887. TYPE: Guatemala. Alta Verapaz: Pansmalá, $1160 \mathrm{~m}$, Mar. 1886, von Türckheim 892 (holotype, US; isotype, $\mathrm{GH}$ ).

Myriocarpa heterostachya Donnell Smith, Bot. Gaz. (Crawfordsville) 13: 29. 1888, nom. illeg., non Myriocarpa heterospicata Donnell Smith.

Discussion. Donnell Smith (1888) sought to correct his hybrid (Latin-Greek) species epithet heterospicata by superfluously publishing Myriocarpa heterostachya Donnell Smith, which has subsequently been widely used in error.

Habitat and distribution. Myriocarpa heterospicata is found in disturbed and undisturbed evergreen wet forest, cloud forest, or oak-pine-liquid amber forest formations from Mexico to Nicaragua at elevations of $100-1800 \mathrm{~m}$. Material from the Atlántida Department in Honduras (Liesner 26318 [BM, MO], Yuncker 8477 [BM, F, MO, NY, US], and Yuncker 8761 [F, NY] is notable for having significantly longer (to $33 \mathrm{~cm}$ ) and more floriferous (to 2400 flowers) pistillate inflorescences than other material from Mesoamerica.

Selected specimens examined. BELIZE. [s. loc.]: Whitefoord 1118 (BM, MEXU, MO). Toledo: Schipp 1033 (BM, F, MO, NY), Gentle 6647 (F, LL). GUATEMALA. [s. loc.]: Skutch 895 (BM, NY, US). Alta Verapaz: Smith 1645 (NY), Standley 70302 (BM, F, US), Standley 70099 (F), Standley 89901 (F, TEX), Steyermark 44187 (F), Steyermark 43919 (F), Tenorio L. et al. 14703 (BM), Tuerckheim 1350 (NY),
Tuerckheim II/1353 (BM, F, MO, NY), Tuerckheim 892 (GH, US), Williams et al. 40159 (F, US), Williams et al. 40201 (BM, F, NY), Williams et al. 43648 (BM, F, US), Wilson 40846 (F), Wilson 40586 (F). Eseuintla: Standley 63385 (F), Standley 89583 (F). Huehuetenango: Steyermark 49507 (F, US). Petén: Contreras 6674 (LL), Contreras 10061 (F, LL), Contreras 3548 (LL), Contreras 10636 (F), Lundell \& Contreras 20104 (LL), Lundell 17759 (LL), Lundell 16261 (F, LL), Smith 6674 (BM), Steyermark 46073 (F, LL), Zomer 133 (F). Quezaltenango: Roe et al. 722 (BM, F). San Marcos: Croat 40799 (MEXU, MO). Suchitepéquez: Standley 66867 (US). HONDURAS. Atlántida: Liesner 26318 (BM), Yuncker et al. 8761 (F, NY), Yuncker et al. 8477 (BM, F, MO, NY, US). Comayagua: Molina R. 10866 (F), Molina R. 5824 (F), Molina R. 5834 (F), Molina R. 10880 (F), Yuncker et al. 6332 (F, MO), Yuncker et al. 5998 (F, MO). Copán: Molina R. \& Molina 30782 (F). MEXICO. [s. loc.]: Breedlove 49023 (MEXU), Matuda 4318 (MEXU, MO), Mejia et al. 43 (MEXU). Chiapas: Breedlove 33068 (F), Breedlove 23847 (MO, NY), Breedlove 57964 (MEXU), Breedlove 33881 (MO), Breedlove 9711 (F, LL), Breedlove \& Thorne 21397 (MEXU, MO, NY), Cabrera \& Cabrera 3886 (MEXU), Croat \& Hannon 65129 (MEXU, MO), Duran \& Levy 440 (MEXU), Hampshire \& Reyes G. 1226 (BM, F), Langman 3878 (BM), Levy \& Durán 318 (MEXU), Martinez 1986 (BM, MO), Matuda 18135 (F, MEXU), Matuda 18080 (F, MEXU), Matuda 4037 (F), Mejia et al. 712 (MEXU), Mendez T. \& Mtz. de López 9492 (MEXU, TEX), Mendez T. et al. 6562 (BM), Mendez T. \& Shilom A. 7177 (BM), Mendez T. et al. 4999 (BM), Mendez T. et al. 5176 (BM), Mendéz T. 6470 (MEXU), Miranda 7697 (MEXU), Miranda 5808 (MEXU), Miranda 5153 (MEXU), Narave F. 1286 (MEXU), Shilom T. 1406 (US), Shilom T. 3643 (MEXU, NY). Tabasco: Magaña \& Zamudio 870 (MEXU), Ventura A. 21482 (MEXU). Veracruz: Cedillo T. 2501 (BM), Nee \& Taylor K. 29414 (BM, NY), Purpus 16265 (F, TEX, US). NICARAGUA. Estelí: Grijalva 960 (BM, MO), Martinez \& Grijalba 1901 (BM), Moreno 19426 (MO). Jinotega: Henrich \& Stevens 235 (BM, MO), Moreno 7694 (BM, MO), Sandino 1021 (BM, MO), Standley 10727 (F), Stevens \& Krukoff 21843 (BM). Matagalpa: Gentry et al. 44042 (MO), Gentry et al. 44063 (MO), Grijalva et al. 3027 (MO), Guzman et al. 886 (BM, MO), Krukoff \& Stevens 23023 (BM, MEXU, MO), Krukoff \& Stevens 23024 (BM, C), Krukoff \& Stevens 23025 (MO), Moreno 17011 (BM, MEXU, MO), Molina R. 22885 (BM, F, MO, NY), Molina R. 30549 (MO), Nee 27659 (BM, MEXU, MO), Neill 829 (BM, MO), Stevens et al. 21313 (BM, MO), Stevens et al. 21312 (BM, MO), Stevens et al. 20327 (BM, MO), Tomlin 22 (BM, MO), Williams et al. 23417 (F, MEXU).

5. Myriocarpa longipes Liebmann, Kongel. Danske Vidensk. Selsk. Skr., Naturvidensk. Math. Afd., ser. 5, 2: 306. 1851. TYPE: Costa Rica. Alajuela: Monte Aguacate, Nov. 1846, Oersted s.n. [5851] (lectotype, designated here, C [100/99-22]; isotype, US).

Myriocarpa inaequilateris Liebmann, Kongel. Danske Vidensk. Selsk. Skr., Naturvidensk. Math. Afd., ser. 5, 2: 307. 1851. TYPE: Costa Rica. Alajuela: Monte Aguacate, Nov. 1846, Oersted 14338 (lectotype, designated here, C [100/99-12], C photo at F). 
Myriocarpa magnifica Rusby, Descr. S. Amer. Pl. 11. 1920. TYPE: Colombia. Magdalena: Santa Marta, near Cacagualita, 460-610 m, 23 Dec. 1898, H. H. Smith 1422 (holotype, NY image seen; isotypes, BM, GH, US).

Myriocarpa yzabalensis (Donnell Smith) Killip, Proc. Biol. Soc. Wash. 40: 29. 1927. Basionym: Myriocarpa longipes Liebmann var. yzabalensis Donnell Smith, Bot. Gaz. (Crawfordsville) 16: 13. 1891. TYPE: Guatemala. Izabal: Monte Cachirulo, $240 \mathrm{~m}$, Apr. 1889, J. Donnell Smith 1644 (holotype, US).

Discussion. The collection selected as lectotype for Myriocarpa longipes was designated a lectotype through an annotation by Dennis Woodland (in sched.) in 1984. This typification was, however, never published. The collection selected as lectotype was made on the basis that it was cited by Liebmann in the original species description and that this was the duplicate with the most fertile material.

The collection selected as lectotype for Myriocarpa inaequilateris was designated a lectotype through an annotation by Dennis Woodland (in sched.) in 1984. This typification was, however, never published. The collection selected as lectotype was made on the basis that it was cited by Liebmann in the original species description.

Habitat and distribution. Myriocarpa longipes is found in disturbed, undisturbed, and secondary evergreen wet forest, essentially streamside or riverside, from sea level to $2400 \mathrm{~m}$, and is distributed from Mexico to South America (Colombia, Bolivia, Brazil).

This species is very variable for leaf shape size and fruit color. Leaf shape varies from ovate to lanceolate, elliptic, or oblanceolate to obovate, encompassing the full range of shapes and dimensions of other Mesoamerican Myriocarpa species. The black shiny fruit cited by Donnell Smith as one of the diagnostic characters for $\boldsymbol{M}$. longipes var. yzabalensis (later raised to specific rank by Killip) appears to be variable within Central American species. For example, it also occurs in some collections of M. heterospicata, and it may be that it is associated with the maturity of the fruit.

Selected specimens examined. [s. loc.]: Oersted A. 14346 (C). BELIZE. [s. loc.]: Schipp 1178 (BM, F, NY), Schipp 8472 (F). Cayo: Gentle 2223 (LL, MEXU, NY, US), Hawkins 1277 (BM). Stann Creek: Dwyer et al. 555 (MO), Gentle 2849 (NY), Gentle 3179 (NY). Toledo: Croat 24329 (MO), Gentle 7178 (LL), Gentle 5288 (LL, MEXU), Gentle 5490 (LL), Whitefoord 1830 (BM), Whitefoord 1582 (BM, MEXU), Stevenson 14494-91 (F), Stevenson 14894-112 (F). COSTA RICA. [s. loc.]: Burger \& Matta U. 4211 (F), Burger \& Matta U. 4748 (NY), Burger \& Matta U. 4657 (F), Leon $606(\mathrm{~F})$, Mier 1926 (C), Mier 1612 (C), Smith 6776 (NY), Valerio 396 (F). Alajuela: Brenes 6568 (F), Brenes 6584 (F, NY), Brenes 6536 (F, NY), Guild \& Herrera 3 (BM), Haber 1523 (MO), Haber ex Bello C. 6427 (MO), Herrera C. 1007 (BM, CR), Herrera C. et al. 429 (BM), Jimenez M. 1719 (F, NY, US),
Molina R. et al. 17405 (F, NY, US), Oersted A. 5851 (F, US), Smith 1423 (MO, NY), Utley \& Utley 3984 (F), Utley \& Utley 3895 (F, MO), Utley \& Utley 3950 (F, MO), Burger et al. 11652 (BM, CR, F, NY). Cartago: Lent 3622 (F), Lems 5055 (NY), Little 20063 (CR), Polakowsky 486 (BM), Taylor 18035 (NY). Guanacaste: Garwood et al. 809 (BM), Grayum \& Herrera 4835 (BM, MEXU), Martin 3727 (BM), Tebbs \& Vickery 1063 (BM, MO). Heredia: Folsom 8784 (F, TEX), Frankie 69c (BM), Grayum 2227A (F), Grayum 2227B (TEX), McDowell 1087 (F), Vargas 272 (BM). Limón: Burger et al. 10333 (NY), Burger \& Liesner 6993 (BM), Antonio 609 (F), Burger \& Antonio 11292 (MEXU), Quiros 506 (F), Robles 1449 (BM), Robles 2652 (BM, CR, F). Puntarenas: Allen 5739 (DS, F), Burger \& Gentry L $8872 A$ (F), Burger \& Swagel 12273 (F), Castro 214 (MO), Castro 203 (MO), Castro 83 (MO), Cordero 163 (MO), Cordero 86 (BM, MO), Delprete 5175 (TEX), Haber \& Zuchowshi 11270 (BM, MO), Haber 1340 (MEXU, MO, TEX), Haber 11020 (MO), Jimenez et al. 1495 (BM, MO), Kernan 17 (BM, CR, F), Lent 3075 (F), Maas et al. 7889 (BM), Raven 20860 (F, MO), Skutch 1947 (CR), Skutch 5379 (CR), Smith 6775 (BM, NY), Tonduz 4826 (BM). San José: Burger \& Barringer, K. 11621 (CR, F), Morales et al. 1126 (BM), Skutch 2320 (MO, NY), Skutch 2321 (NY), Téllez et al. 4228 (MEXU), Williams et al. 28451 a (F, NY), Zamora \& Carlson 419 (MEXU). GUATEMALA. Alta Verapaz: Croat 41595 (MO, US), Croat 41594 (MO), Choc \& Stewart 36 (CAS), Goll 223 (NY). Chimaltenango: Standley 80161 (F). El Quiché: Ventur 227 (F). Izabal: Contreras 11544 (LL), Johnson 1262 (US), Kellerman 7319 (NY, US), Kellerman s.n. [125402] (MEXU), Smith 1644 (US), Stevens et al. 25498 (BM), Steyermark 41791 (US), Steyermark 41824 (F), Steyermark 39952 (F, US), Steyermark 38263 (F), Steyermark 39091 (F), Steyermark 41840 (F). Petén: Contreras 6630 (F, LL), Contreras 9298 (LL), Tun 0. s.n. [US 2735523] (US), Tun O. 1489 (BM). Quetzaltenango: Standley 67218 (F), Standley 85087 (F), Standley 65355 (F). Sacatepéquez: Standley 88993 (F). San Marcos: Croat 40801 (MEXU, MO), Dwyer 14403 (BM, MO), Standley 86275 (F). HONDURAS. [s. loc.]: Wilson 253 (NY). Atlántida: Barkley \& Hernández M. 40035 (TEX), Croat \& Hannon 64621 (BM, MO), Liesner 26401 (BM), Standley 52953 (F), Standley 54061 (US), Standley 54626 (F). Comayagua: Edwards P-454 (F), Molina R. 10843 (F, NY). Gracias a Dios: Saunders 1193 (NY). Yoro: Hazlett 3157 (BM, F, MEXU). MEXICO. [s. loc.]: Hanan 290 (MEXU), Juzepczuk 1925-26 (F), Mendez T. 9581 (MEXU). Chiapas: Bossé 8474 (F), Breedlove 11085 (LL), Breedlove \& Smith R. 22132 (MEXU, MO), Breedlove 14922 (LL, US), Breedlove 23969 (LL, MO), Breedlove 8844 (F, LL), Breedlove 24226 (MEXU), Breedlove 21924 (LL), Brett 280 (TEX), Burnham 10 (BM), Chavelas P. et al. 691 (MEXU), Chavelas P. et al. 1286 (MEXU), O. F. Clarke 224 (NY), Croat \& Hannon 65220 (BM), Flores C. \& Espejo 546 (MEXU), Fuentes s.n. "Ene 24 1955, 50270" (MEXU), Hernández G. 705 (LL), Hoover 140 (MO), Juzepczuk 1805 (F), Laughlin 287 (F, LL), Mendez T. et al. 5470 (BM), Mendez G. 8052 (BM), Martinez S. 17054 (BM, MEXU), Martinez S. 17805 (BM), Martinez S. et al. 3138 (BM, MEXU), Sánchez s.n. "9 Nov 1977" (TEX), Shilom T. 2906 (LL), Spellman et al. 193 (MO), Ventura L. 2842 (MEXU). Tabasco: Cowan \& Zamudio 3361 (MO, NY), Croat et al. 65348 (MEXU), Martinez S. 34708 (BM). Veracruz: Fay \& Hernandez 829 (NY, US), Gentry et al. 32306 (MO), Hernandez O. 50 (MEXU), Ibarra M. 2267 (MO), Ibarra M. 789 (MO), Martinez C. 1745 (MO, NY), Nee \& Taylor 29888 (MO, NY), Perino \& Perino 3102 (NY), Sinaca C. 800 (MO), Soto \& Horvitz 47 (NY), Williams 8559 (US), Williams 8403 (US), Vázquez Y. 
411 (MO). NICARAGUA. [s. loc.]: Baker 72 (DS, MO, NY), Englesing 64 (F), Tate 392 (BM). Boaco: Moreno 20196 (BM, MO), Moreno 20275 (MO). Chinandega: Shimek \& Smith 4 (F). Chontales: Gentry et al. 43949 (BM, MO), Grijalva \& Bradford 3643 (BM, MO, NY), Sandino 5103 (BM, MO), Stevens \& Krukoff 6147 (BM, MO), Vincelli 96 (BM, MO). Jinotega: Atwood et al. 6899 (F, MO), Stevens \& Krukoff 16625 (BM, MO), Stevens \& Krukoff 16460 (BM, MO). Managua: Garnier A. 346 (US), Grant 1046 (US). Masaya: Standley 8714 (F), Standley 8733 (F). Matagalpa: Araquistain \& Moreno 2721 (BM, MO), Araquistain \& Moreno 2579 (MO), Castro 2272 (BM, MO), Stevens \& Krukoff 11880 (BM, MEXU, MO), Stevens et al. 21212 (BM, MO), Stevens et al. 21211 (MO), Stevens et al. 20977 (BM, MO), Williams et al. 23845 (NY). Río San Juan: Moreno 23221 (BM, MO), Neill \& Vincelli 3577 (BM, MO), Neill 3343 (BM, MEXU, MO). Rivas: Moreno 19646 (MO), Sandino 4113 (BM, MO), Stevens \& Krukoff 6600 (BM, MO). Zelaya: Krukoff \& Stevens 18787 (BM, MO), Krukoff \& Stevens 6302 (BM, MO), Little 25324 (MO, US), Moreno 15000 (BM, MO), Moreno 19128 (BM, MO), Moreno 14762 (BM, MEXU, MO), Moreno \& Robleto 20666 (BM, MO), Moreno \& Sandino 14965 (BM, MO), Neill 4135 (MO), Ortiz 546 (MO), Ortiz 627 (BM, MO), Ortiz 745 (BM), Ortiz 1500 (BM, MO), Ortiz 573 (MO), Ortiz 1654 (BM, MO), Ortiz 906 (MO), Ortiz 1538 (BM, MO), Pipoly 4712 (BM, MO), Pipoly 5895 (MO), Pipoly 3532 (BM, MO), Pipoly 4957 (MO), Pipoly 6114 (BM, MO), Pipoly 4864 (BM, MO), Pipoly 3752 (BM, MO), Pipoly 4607 (BM, MO), Stevens \& Krukoff 6813 (BM, MO), Stevens \& Krukoff 12660 a (MO), Stevens \& Krukoff 12057 (BM, MEXU, MO), Sandino 2411 (MO), Stevens \& Krukoff 12568 (BM, MO), Stevens \& Krukoff 13085 (MO), Stevens \& Krukoff 6895 (BM, MO), Stevens \& Krukoff 13052 (BM, MEXU, MO), Stevens \& Krukoff 19384 (BM, MO), Stevens \& Krukoff 6828 (BM, MO), Vincelli 239 (BM, MO), Vincelli 137 (BM, MO), Vincelli 205 (BM, MO). PANAMA. [s. loc.]: Cowell 113 (NY), Piper 5841 (C, US), Seemann 1575 (BM). Bocas del Toro: Correa et al. 3396 (F), Dunlap 300 (F), Oliver 950 (NY), McPherson 6763 (MEXU, MO, TEX), Proctor 414 (F, NY), Wedel 1692 (MO), Wedel 1411 (MO), Wedel 1505 (MO). Chiriquí: D'Arcy et al. 13009 (BM), Davidson 488 (F); Pittier 5157 (NY, US), Stevens et al. 18432 (BM), Terry \& Terry 1646 (F). Coclé: Allen 4218 (US), Dwyer 11852 (NY), Hamilton \& Davidse 2790 (MEXU, MO), Sytsma \& D'Arcy 3622 (MEXU, MO). Colón: Churchill W. \& de Nevers 4232 (MEXU), Gentry 6727 (MO), Antonio 3334 (LL), Salazar 44 (F), Pittier 2487 (BM, NY), Knapp \& Schmalzel 3603 (LL), Antonio 3834 (BM), Nee 7146 (US). Darién: D'Arcy \& McPherson 16219 (MEXU), D'Arcy \& Sytsma 14464 (MO), Garwood et al. 27 (BM), Knapp \& Mallet 3038 (LL), McDonagh 520 (BM), Nee 9110 (LL), Whitefoord \& Eddy 137 (BM), Whitefoord \& Eddy 196 (BM), Reveal \& Duke 4860 (MO). Kuna Yala: Bangham 586 (F), D'Arcy 13651 (BM, MEXU), Hamilton \& Stockwell 2941 (MEXU, MO). Panamá: Aviles 27 (F), Brown 47 (F), Brown 108 (F), Busey 313 (MO, NY), Croat 4196 (NY), Dodge et al. $16997 a$ (BM, F, NY), Fendler 280 (BM, F, MO), Folsom et al. 6846 (MO), Fosberg R. 55630 (NY), Garwood 2089a (BM), Gentry 6667 (F, NY), Hadick 79 (MO), Hadick 24 (MO), Mendez 191 (F), Shattuck 242 (F), Standley 27572 (US), Smith \& Smith 3309 (F, MEXU, US), Sytsma \& D'Arcy 3691 (LL), Tyson et al. 4484 (MO), van der Werff \& Herrera 6189 (MEXU, MO), Wetmore \& Abbe 14 (F), Williams 728 (NY), Woodworth \& Vestal 309 (F), Zetek 1-1926 (F).

6. Myriocarpa obovata Donnell Smith, Bot. Gaz. (Crawfordsville) 46: 117. 1908. TYPE: Hon- duras. Santa Bárbara: San Pedro Sula, 800 m, 17 Jan. 1887, C. Thieme 5500 (holotype, US).

Myriocarpa paniculata S. F. Blake, Contr. Gray Herb. 52: 61. 1917. TYPE: Nicaragua. Granada: Volcán Mombacho, 20 Feb. 1903, C. F. Baker 2489 (holotype, GH; isotypes, BM, CAS, DS, F, MO, US).

Habitat and distribution. Myriocarpa obovata is found in semi-evergreen and evergreen disturbed and undisturbed tropical moist forest, from sea level to $1400 \mathrm{~m}$, and is distributed from Mexico to Costa Rica.

A few collections from Guatemala and Oaxaca (Mexico) (Caldéron 1360 [NY], Williams et al. 40159 [US], and Williams et al. 40201 [BM, F, NY]) have minutely pitted adaxial leaf surfaces as opposed to the usual smooth surface. These minute pits are characteristic of Myriocarpa heterospicata and M. trifurca, but their function and homology to other structures (e.g., glands or hairs) are unknown.

Selected specimens examined. BELIZE. [s. loc.]: Schipp 869 (BM, F, MO, NY). Cayo: Cowan et al. 5155 (MEXU), Gentle 2212 (F, LL, MEXU, NY, US), Monro et al. 1117 (BM, MEXU), Spellman 1376 (MO). COSTA RICA. San José: Cascante 194 (CR, F), Hammel \& Morales 20610 (CR, MO), Jimenez M. et al. 748 (BM, CR, F, MO), Jiménez M., Q 450 (F). EL SALVADOR. Ahuacapán: Chinchilla \& López s.n. [ISB247] (F), Guerrero s.n. [ISF00211] (LAGU, MO), Sandoval \& Sandoval 296 (F, LAGU), Sermeno 132 (BM, LAGU), Standley 19780 (NY). La Libertad: Calderón 1514 (NY). GUatemala. Petén: Contreras 4094 (F, LL, MEXU), Contreras 7384 (F, LL), Contreras 650 (F, LL), Contreras 1927 (F, LL), Lundell 15832 (F, LL), Lundell 15250 (F, LL), Lundell 2194 (F), Lundell 15421 (F, LL, MEXU), Molina R. 15823 (F). HONDURAS. [s. loc.]: Record \& Kuylen 9974 (NY, US), Record \& Kuylen H. 28 (US). Colón: Evans 1098 (BM). Cortés: Allen 6471 (F), Molina R. 3604 (F). Olancho: Blackmore \& Heath 2033 (BM, MEXU), Croat \& Hannon 64131 (BM, MEXU, MO). Santa Bárbara: Evans 1004 (BM), Thieme 5500 (US). Yoro: Croat \& Hannon 64662 (BM, MEXU, MO). MEXICO. Chiapas: Breedlove 38249 (MEXU), Breedlove 34892 (MEXU), Breedlove 33205 (MEXU), Mendez T. 6107 (MEXU), Mendez T. 5891 (BM, MEXU), Mendez T. 4255 (BM, MEXU). Oaxaca: Calderón 1360 (MEXU, NY), Campos V. \& Torres 1596 (BM), Torres C. \& Cortes A. 8842 (MEXU). Tabaseo: Hanan 469 (MEXU). Veracruz: Dorantes 2991 (MO), Wendt et al. 3229 (MO), M. Vázquez T. et al. 2480A (MO). NICARAGUA. Chontales: Stevens \& Krukoff 6105 (BM, MO), Vincelli 94 (MO). Granada: Baker 854 (US), Blake 2489 (BM, CAS, DS, F, GH, MO, US), Grijalva et al. 2889 (BM, MEXU, MO), Moreno 6328 (BM, MO), Moreno 6307 (BM, MO), Moreno 6385 (BM, MO), Moreno 6449 (BM, MO), Moreno 15949 (BM, MO). Madriz: Williams \& Molina R. 20247 (F, NY). Managua: Garnier 506 (US), Garnier 1736 (US), Grant 952 (BM, F), Grant 1093 (US). Masaya: Standley \& Antonio G. 8108 (F). Matagalpa: Castro 2375 (MO), Castro 2355 (MO), Krukoff \& Stevens 11837 (BM, MO), Moreno 25418 (BM, MO), Moreno 23568 (MO), Stevens et al. 21361 (MO). Rivas: Moreno 19813 (BM, MO), Moreno 19607 (MO), Neill \& Vincelli 3218 (BM, MO), Robleto 927 (BM, MO), Robleto 338 (BM, MO), Robleto 147 (BM, MO), Sandino 4149 (MO), Sandino 4264 (MO), Sandino 4259 (MO), Sandino 566 (BM, MO), Stevens \& Krukoff 6592 (BM, MO). Zelaya: Neill 4302 (BM, MO), Ortiz 
1805 (BM, MO), Pipoly 4394 (BM, MO), Stevens \& Krukoff 6880 (BM, MO),

7. Myriocarpa trifurea A. K. Monro, sp. nov. TYPE. Mexico. Veracruz: ca. $4 \mathrm{~km}$ al $\mathrm{N}$ de Xalapa, por la carretera a México, ladera exposado $\mathrm{N}$ del Río Sedeño, $1450 \mathrm{~m}, 1$ Mar. 1981, A. Rebolledo V. 302 (holotype, F; isotype, NY). Figure 2.

Species nova Myriocarpae heterospicatae Donnell Smith similis, sed ab ea lamina supra efoveata, cystolithis radialiter dispositis atque inflorescentiae staminalis ramulis minoribus differt.

Shrub or tree to $6 \mathrm{~m}$, evergreen. Young shoots pubescent to densely pubescent, the hairs to $0.5 \mathrm{~mm}$, appressed or weakly appressed, straight to curved, internodes 7-17 × 2.5-4 mm. Stipules 6.5-10 mm; petioles 17-62 × 1-1.5 mm, pubescent to densely pubescent, the hairs $0.25-0.75 \mathrm{~mm}$; leaf blade $100-$ $185 \times 41-110 \mathrm{~mm}$, elliptic, frequently asymmetrically so, membranous, subchartaceous to chartaceous; adaxial surface not minutely pitted, pubescent, the hairs 1-1.25 mm, crooked, straight, or weakly curved, the cystoliths fusiform, V-shaped fusiform, and Yshaped fusiform, appearing flattened at their margins, arranged radially around hair bases and/or glands and perpendicular to veins; abaxial surface pubescent to densely pubescent, the hairs $0.25-0.75 \mathrm{~mm}$, appressed, weakly curved or straight, cystoliths absent; principal veins 3 , principal lateral veins not prominent, visible for $1 / 3-1 / 2$ of the leaf length, secondary veins 3 to 5 pairs, forming a $45^{\circ}-60^{\circ}$ angle with midrib, domatia present in axils of primary and secondary veins; base cuneate, acute, or obtuse; margins serrate to base; apex cuspidate. Staminate inflorescences $42-85 \mathrm{~mm}$, with 2 or 3 orders of branching, with a total flower-bearing branch length of 69-102 mm; peduncular bracts ca. $1.5 \mathrm{~mm}$; peduncle densely pubescent, the hairs ca. $0.5 \mathrm{~mm}$; flowers subsessile or sessile. Pistillate inflorescences 3 to 14 per stem, pendent to spreading, 45-240 $\times 2.5-$ $3.25 \mathrm{~mm}$, bearing 707 to $1204(5600)$ flowers, green or green-brown when fresh, pale brown when dry, trichotomously and/or dichotomously branched 1 or $2 \times$, with a total flower-bearing branch length of 108$500 \mathrm{~mm}$ and flower density of 52 to 112 per $\mathrm{cm}$; peduncular bract $1.5-9 \mathrm{~mm}$; peduncle $8-30 \mathrm{~mm}$, pubescent or sparsely pubescent, the hairs 0.38 $0.5 \mathrm{~mm}$, appressed or erect, weakly curved, crooked, or straight; flowers/fruit subsessile to sessile, pedicels when present ca. $0.13 \mathrm{~mm}$. Staminate flowers ca. 0.68 $\times 1.75-2 \mathrm{~mm}$ immediately prior to anthesis; tepals immediately prior to anthesis ca. $1.5 \mathrm{~mm}$; stamens not seen. Pistillate flowers/fruit 1.25-1.5 × 0.38-0.5 mm; tepals $2,0.5-0.675 \mathrm{~mm}$, weakly appressed; ovary
$0.68-0.75 \mathrm{~mm}$, elliptic or ovate, constricted from base for $1 / 4-1 / 3$ ovary length, pubescent, the hairs 0.13 $0.5 \mathrm{~mm}$; style ca. $0.5 \mathrm{~mm}$; stigma asymmetrical.

Habitat and distribution. Myriocarpa trifurca is found in pine-oak forest formations, wet forest, and riparian and secondary forest at elevations of 100$1600 \mathrm{~m}$ in southern Mexico (Chiapas and Veracruz).

IUCN Red List category. Conservation status for Myriocarpa trifurca must be considered as Least Concern (LC) according to IUCN Red List criteria (IUCN, 2001), owing to the fact that the species has been collected 34 times in several localities in Chiapas and Veracruz.

Etymology. The species is named for the fact that the pistillate inflorescences are frequently trichotomously branched, a relatively uncommon branching arrangement within Myriocarpa.

Discussion. Collections of Myriocarpa trifurca are most frequently determined as $\boldsymbol{M}$. longipes or $\boldsymbol{M}$. heterostachya $(=$ M. heterospicata $)$. Myriocarpa trifurca is most similar to $M$. heterospicata because of its frequently short staminate inflorescences and elliptic leaves, and to $M$. cordifolia because of its broad pistillate inflorescence branches. The three species may be distinguished from each other as follows: (1) in M. cordifolia, adaxial leaf surface not minutely pitted, cystoliths radially arranged around hair bases and glands; staminate inflorescence $90-310 \mathrm{~mm}$; pistillate inflorescence branches $3.5-5.5 \mathrm{~mm}$ diam.; pistillate flowers/fruit $1.75-2.25 \mathrm{~mm}$; (2) in M. heterospicata, adaxial leaf surface minutely pitted, cystoliths randomly scattered; staminate inflorescence 25$170 \mathrm{~mm}$; pistillate inflorescence branches 1.75$3 \mathrm{~mm}$ diam.; pistillate flowers/fruit $1-2 \mathrm{~mm}$; and (3) in $M$. trifurca, adaxial leaf surface not minutely pitted, cystoliths radially arranged around hair bases and glands; staminate inflorescence $42-85 \mathrm{~mm}$; inflorescence branches $2.5-3.3 \mathrm{~mm}$ diam.; pistillate flowers/ fruit $1.25-1.5 \mathrm{~mm}$.

Paratypes. MEXICO. Chiapas: Mun. Pueblo Nuevo Solistahuacán near Clinica Yerba Buena, $3 \mathrm{~km} \mathrm{NW}$ of Pueblo Nuevo Solistahuacán, $1700 \mathrm{~m}, 11$ July 1972, D. E. Breedlove 26038 (LL); Mun. Ocosingo, $15 \mathrm{~km} \mathrm{SE}$ of Ocosingo on rd. to San Cristóbal de Las Casas, 1372 m, 1 Sep. 1981, D. E. Breedlove 52593 (LL); Mun. Ocosingo, $70 \mathrm{~km} \mathrm{SW} \mathrm{of}$ Palenque on rd. to Ocosingo along the Jol Uk'um, $550 \mathrm{~m}, 4$ Dec. 1980, D. E. Breedlove \& F. Almeda 48404 (LL); Mun. Mapastepec, Reserva El Triunfo poligono $1,15^{\circ} 39^{\prime} \mathrm{N}$, $92^{\circ} 48^{\prime} \mathrm{W}, 1450 \mathrm{~m}, 17$ June 1990 , M. Heath \& A. Long 1074 (MEXU); Mun. Pueblo Nuevo Solistahuacán, along the ridge above Pueblo Nuevo Solistahuacán, 2000 m, 15 Aug. 1967, A. Shilom T. 2904 (LL). Veracruz: carretera TeoceloIxhuacan de los Reyes, Ixhuacan de los Reyes, 1610 m, 12 Mar. 1976, S. Avendano R. et al. 181 (MO, NY); al lado N de Ejido La Perla de San Martín, ca. 15 km al N de Catemaco, 


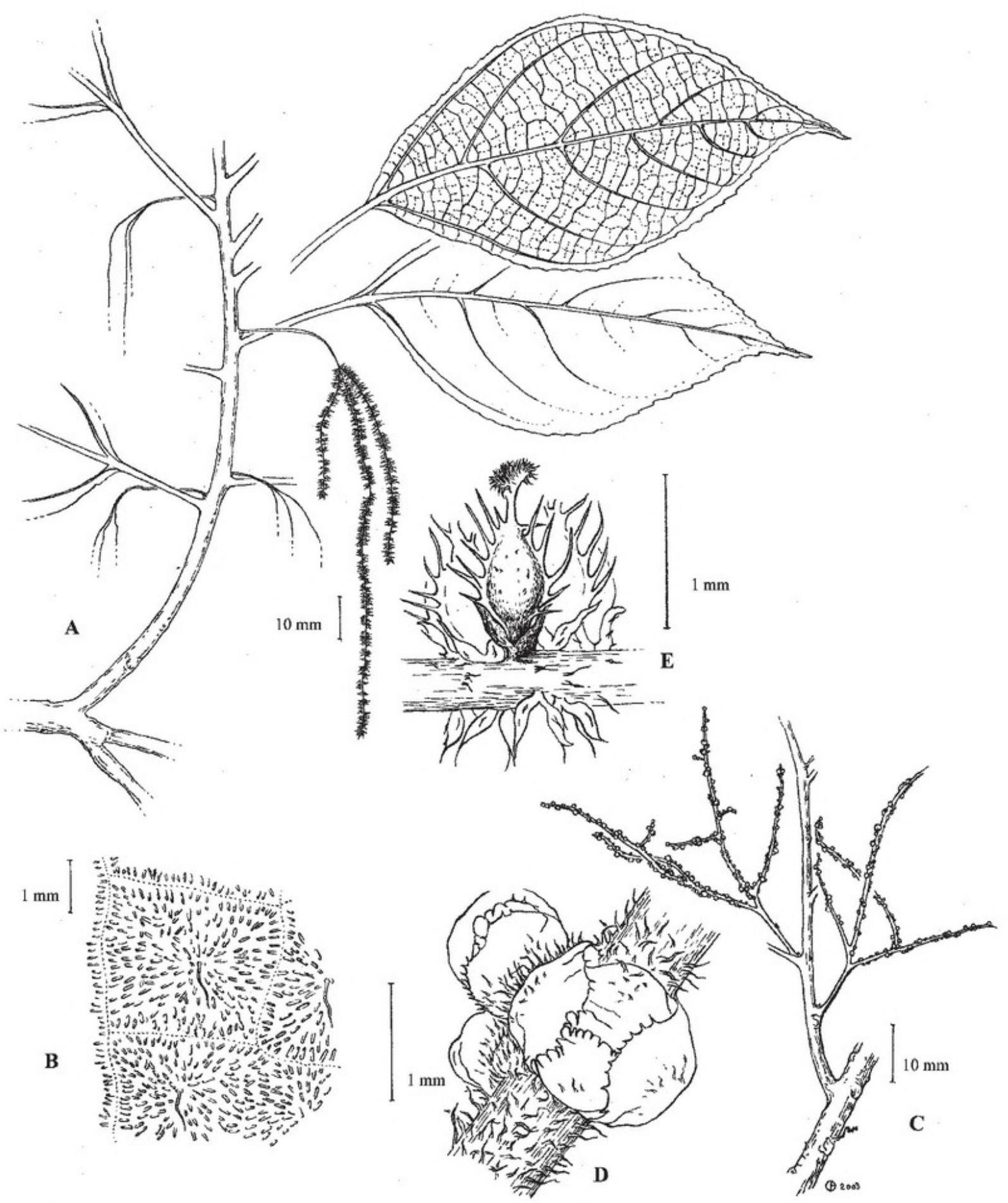

Figure 2. Myriocarpa trifurca A. K. Monro. - A. Habit, with pistillate inflorescences. -B. Cystolith arrangement of adaxial leaf surface. - C. Staminate inflorescence. -D. Staminate flowers immediately prior to anthesis. -E. Pistillate flowers. A-E drawn from the holotype, A. Rebolledo V. 302 (F).

Catemaco, $18^{\circ} 31^{\prime} \mathrm{N}, 95^{\circ} 05^{\prime} \mathrm{W}, 700 \mathrm{~m}, 2$ Mar. 1972, J. H. Beaman 5826 (NY, TEX); beche de la Martinica a $2 \mathrm{~km}$, Banderilla rancho del Sr. González Parra, Mun. Banderilla, $19^{\circ} 35^{\prime} \mathrm{N}, 96^{\circ} 57^{\prime} \mathrm{W}, 1480 \mathrm{~m}, 11$ Nov. 1977, G. Castillo C. et al. 124 (NY); carretera Xalapa-Naolinco, zonas cafetaleras, Jilotepec, 1400 m, 2 Mar. 1976, C. Hernández A. et al. 54 (BM, MO); Jardín Botánico, Estación de Biología Tropical Los Tuxtlas, $18^{\circ} 34-36^{\prime} \mathrm{N}, 95^{\circ} 04-09^{\prime} \mathrm{W}, 200 \mathrm{~m}, 20$ July $1983, G$. Ibarra M. 739 (MO); Estación de Biología Tropical Los Tuxtlas, $18^{\circ} 34-36^{\prime} \mathrm{N}, 95^{\circ} 04-09^{\prime} \mathrm{W}, 160 \mathrm{~m}, 15$ May $1984, G$. Ibarra M. 1664 (MO); Estación de Biología Tropical Los
Tuxtlas, $18^{\circ} 34-36^{\prime} \mathrm{N}, 95^{\circ} 04-09^{\prime} \mathrm{W}, 160 \mathrm{~m}, 13$ Dec. $1983, G$. Ibarra M. 1123 (MO); Estación de Biología Tropical Los Tuxtlas, $18^{\circ} 34-36^{\prime} \mathrm{N}, 95^{\circ} 04-09^{\prime} \mathrm{W}, 160 \mathrm{~m}, 25 \mathrm{Jan} .1984, G$. Ibarra M. 1120 (MO); Estación de Biología Tropical Los Tuxtlas, $18^{\circ} 34-36^{\prime} \mathrm{N}, 95^{\circ} 04-09^{\prime} \mathrm{W}, 200 \mathrm{~m}, 3$ Apr. 1984, G. Ibarra M. 1453 (MO); Mun. Orizaba, Cerro San Cristóbal, frente a Orizaba, $1400 \mathrm{~m}, 17$ Dec. 1970, A. Lot 1105 (BM, MEXU not seen, TEX, XAL not seen); colonia militar Balzapote, San Andres Tuxtla, $100 \mathrm{~m}, 28$ July 1970, G. Martínez C. 3115 (MO); gorge of Río Metlac, just downstream and beneath the bridges of the Córdoba-Orizaba hwy., $150 \mathrm{~m}$ 
above Cuota México, $1 \mathrm{~km}$ (by air) W of Fortín, Mun. Iztaczoquiatlán, $18^{\circ} 54^{\prime} \mathrm{N}, 97^{\circ} 00^{\prime} 30^{\prime \prime} \mathrm{W}, 900 \mathrm{~m}, 7$ Feb. 1984 , M. Nee \& K. Taylor 29414 (BM, MO, NY); along very winding rd. from Naolinco to Misantla, $13 \mathrm{~km}$ by rd. $\mathrm{S}$ of turnoff to Yecuatla and $6 \mathrm{~km}$ by rd. $\mathrm{N}$ of Paz de Enríquez, Mun. Yecuatla, $19^{\circ} 51^{\prime} \mathrm{N}, 96^{\circ} 48^{\prime} 30^{\prime \prime} \mathrm{W}, 1200 \mathrm{~m}, 2$ Apr. 1983, $M$. Nee et al. 26354 (NY); $2 \mathrm{~km}$ al NE de Iztapan, camino a Dos Pozitos, Tonayan, $1200 \mathrm{~m}, 28$ Nov. 1975, R. Ortega et al. 32 (MO); ravines near Orizaba, $4000 \mathrm{ft}$., $18 \mathrm{Jan} .1895, C$. G. Pringle 6093 M (BM, US); Zacuapan, Jan. 1905, C. A. Purpus 2964 (BM, US); on rocks, barranca near La Palmilla, 1934, $C$. A. Purpus 16265 (F, US); el brincadero, cerca de un arroyo que atraviesa el camino de Jilotepec a Naolinco, 28 Dec. 1975, C. H. Ramos 354 (US); ca. 4 km al N de Xalapa por la carretera a México, ladera exposición N del Río Sedeño, 1450 m, I Mar. 1981, A. Rebolledo V. 302 (MO, NY); Apatlanco $2 \mathrm{~km} \mathrm{~N}$ de Santa Ana Atzacan, $1300 \mathrm{~m}, 4$ Jan. 1967, M. Rosas R. \& R. Hernandéz 130 (BM); Cerro de Escamela al N de Orizaba, $1450 \mathrm{~m}, 17$ Jan. 1968, M. Rosas R. 989 (BM); Cerro de Chicahuaxtla-Cuauhtlapan, $1600 \mathrm{~m}$, 22 Jan. 1968, M. Rosas R. 1041 (BM); Cerro de San Cristóbal, $5 \mathrm{~km}$ al SW de Orizaba, 1300-1600 m, 15 Dec. 1959, J. Rzedowski 12171 (MO); Cerro Lázaro Cárdenas lote 71, Estación de Biología Tropical Los Tuxtlas, $18^{\circ} 34-36^{\prime} \mathrm{N}$, 95 04-09'W, 600 m, 9 Aug. 1985, S. Sinaca C. 186 (MO); limites con Omelca, Mun. Coetzala, $600 \mathrm{~m}, 16$ July 1976, V. Vázquez T. 469 (NY); Tonacalco, Zongolica, $1150 \mathrm{~m}, 4$ Mar. 1976, C. Velázquez L. 22 (MO); Mun. Teocelo, La Barranca, 1000 m, 22 Feb. 1979, F. Ventura A. 15799 (TEX); Mun. Teocelo, Barranca de Teocelo, 1020 m, 23 Dec. 1975, M. G. Zola B. 116 (NY).

Acknowledgments. I am grateful to Norman Robson (BM) for help with the Latin diagnoses; Cornelia Blick for the illustrations; the curators at BM, C, F, GH, INB, K, LL, MEXU, MO, NY, P, PMA, TEX, and US for access to collections; to Bob Press for comments on the manuscript; and to Roy Gereau and an anonymous reviewer for their invaluable reviews.

\section{Literature Cited}

Bentham, G. 1846. The Botany of the Voyage of H.M.S. Sulphur, Pt. 6: 168, t. 55.

Burger, W. 1977. Myriocarpa, Flora Costaricensis. Fieldiana, Bot. 40: 237-240.

Davidse, G., M. Sousa S. \& A. O. Chater. 1994. Introducción General. Flora Mesoamericana, Vol. 6: Alismataceae a Cyperaceae. Universidad Nacional Autónoma de México, México, D.F.; Missouri Botanical Garden, St. Louis; The Natural History Museum, London.

Donnell Smith, J. 1888. Undescribed plants from Guatemala II. Bot. Gaz. (Crawfordsville) 13: 26-29.

Friis, I. 1989. The Urticaceae: A systematic review. Pp. 285-308 in P. R. Crane \& S. Blackmore (editors), Evolution, Systematics, and Fossil History of the Hamamelidae, Vol. 2. Systematics Association Special Volume 40B. Oxford Science Publications, Oxford, United Kingdom.

International Plant Names Index (IPNI). 2006. Available at <http://www.ipni.org>, accessed 1 July 2006.

IUCN. 2001. IUCN Red List Categories and Criteria, Version 3.1. Prepared by the IUCN Species Survival Commission. IUCN, Gland, Switzerland, and Cambridge, United Kingdom.

Monro, A. K. 2006. The revision of species-rich genera: A phylogenetic framework for the strategic revision of Pilea (Urticaceae) based on cpDNA, nrDNA, and morphology. Amer. J. Bot. 93: 426-441.

Standley, P. C. \& J. A. Steyermark. 1952. Pilea, Flora of Guatemala. Fieldiana, Bot. 3: 404-406.

Weddell, H. A. 1856. Urera. Pp. 484-490 in G. Baudry \& J. Baudry (editors), Monographie de la famille des Urticacées. G. \& J. Baudry, Paris.

1869. Myriocarpa. In A. de Candolle (editor), Prodr. (DC.) $16,235^{33}-235^{36}$. Paris. 


\section{$2 \mathrm{BHL}$ Biodiversity Heritage Library}

Monro, Alexandre K. 2009. "Two New Species and a Nomenclatural Synopsis of Myriocarpa (Urticaceae) from Mesoamerica." Novon a journal of botanical nomenclature from the Missouri Botanical Garden 19, 85-95.

View This Item Online: https://www.biodiversitylibrary.org/item/124658

Permalink: https://www.biodiversitylibrary.org/partpdf/121883

\section{Holding Institution}

Missouri Botanical Garden, Peter H. Raven Library

\section{Sponsored by}

Missouri Botanical Garden

\section{Copyright \& Reuse}

Copyright Status: Permission to digitize granted by rights holder Rights: https://www.biodiversitylibrary.org/permissions

This document was created from content at the Biodiversity Heritage Library, the world's largest open access digital library for biodiversity literature and archives. Visit BHL at https://www.biodiversitylibrary.org. 